\title{
Urine synaptopodin excretion is an important marker of glomerular disease progression
}

\author{
Soon Kil Kwon, Seung Jung Kim, and Hye-Young Kim
}

Renal Division, Department of Internal Medicine, Chungbuk National University College of Medicine, Cheongju, Korea

Received: July 18, 2015

Revised : April 9, 2016

Accepted: June 21, 2016

\section{Correspondence to}

Soon Kil Kwon, M.D.

Renal Division, Department of Internal Medicine, Chungbuk National University College of Medicine, 1 Chungdae-ro, Seowon-gu, Cheongju 28644, Korea

Tel: $+82-43-269-6020$

Fax: $+82-43-273-3252$

E-mail: kwon@chungbuk.ac.kr
Background/Aims: Podocytes play an important role in maintaining the glomerular filtration barrier and in formation of the slit diaphragm. Podocyte loss is associated with chronic kidney disease progression, but it is not clear whether urinary podocyte proteins in urine reflect the clinical extent of glomerular damage. We investigated the correlation between the amounts of urinary podocyte proteins and renal function and albuminuria.

Methods: The study enrolled 33 patients with diabetic kidney disease or glomerular disease and measured urinary podocytes proteins using Western blotting. Urinary podocyte proteins were measured according to the density of the bands on Western blotting. We measured serum creatinine and the spot urine albu$\mathrm{min} /$ creatinine ratio as markers of renal damage, and compared the correlation of urinary podocyte protein in the glomerular disease patients.

Results: The mean patient age was $49.3 \pm 16.5$ years, the mean serum creatinine level was $2.30 \pm 1.76 \mathrm{mg} / \mathrm{dL}$, and the mean albumin/creatinine ratio was $4.85 \pm 3.52$. Among the podocyte proteins, urine synaptopodin showed strong correlation with serum creatinine by multivariate regression analysis $(p<0.001)$ and showed linear correlation $(r=0.429, p<0.01)$. Urine podocyte proteins were increased in patients with diabetes, and synaptopodin showed the greatest significant difference $(7.68 \pm 5.61$ vs. $2.56 \pm 3.11, p<0.001)$, but this might be associated with renal impairment. The urine albumin excretion did not differ between the diabetics and non-diabetics $(p=0.73)$.

Conclusions: Urine synaptopodin is associated with serum creatinine elevation in the patients with glomerulonephritis including diabetic kidney disease regardless of urine albumin excretion. We suggest that the urine synaptopodin level can predict glomerular damage independently of the urine albumin excretion.

Keywords: Glomerulonephritis; Podocytes; Synaptopodin; Proteinuria; Kidney injury

\section{INTRODUCTION}

Chronic kidney disease is a spectrum of pathophysiologic processes with serum creatinine elevation and tubular atrophy with glomerulosclerosis [1]. Chronic inflammation of glomeruli spreads to the tubules and interstitium in association with proteinuria, and chron- ic volume overload makes intraglomerular hypertension, mechanical and chemical glomerular injury leads to increase amount of proteinuria [2]. Although proteinuria is a marker of glomerular disease progression, some of membranous nephropathy and most minimal change disease have excellent prognosis even with heavy proteinuria. 
Podocytes play an important role as a glomerular filtration barrier. In diabetic nephropathy, podocyte detachment and the loss of endocapillary fenestration cause proteinuria and progressive glomerular injury [3]. Consequently, urinary podocyte loss might be a better predictor of glomerular damage than proteinuria in glomerulonephritis [4]. The changes in the podocyte slit diaphragm result in proteinuria in most forms of glomerulonephritis. Slit diaphragm proteins such as nephrin, podocin, and podocalyxin are associated with the proteinuria seen in various kidney diseases, including diabetic nephropathy. When glomerular damage occurs, the slit diaphragm is destroyed and the urinary excretion of slit diaphragm proteins might serve as a marker of diabetic kidney disease progression [5].

Synaptopodin is an actin-associated podocyte protein, which is important for the integrity of the actin cytoskeleton and regulates podocyte cell migration [6]. Synaptopodin is associated with the anti-proteinuric effect of cyclosporine in focal segmental glomerulosclerosis [7]. However, little is known about association between synaptopodin and advanced glomerular injury including diabetic kidney disease progression.

We investigated to use synaptopodin as a urinary biomarker which could assume podocyte damage or deletion in advanced glomerular disease, and to compare with other important slit diaphragm proteins in urine. Also, we compared the urinary excretion of synaptopodin and slit diaphragm in the patients with glomerular disease which show large amount of proteinuria to evaluate podocyte damage in advanced glomerular disease based on their correlation with serum creatinine levels or amount of proteinuria.

\section{METHODS}

\section{Patients}

The study included 33 patients with glomerular diseases including diabetic chronic kidney disease and primary glomerular disease who had been followed regularly in our outpatient department. All the patients had proteinuria $>1.0 \mathrm{~g} /$ day. The glomerulonephritis included various kinds of primary and secondary glomerulonephritis, such as minimal change disease, membranous nephropathy, focal segmental glomerulosclerosis, im- munoglobulin A (IgA) nephropathy, and lupus nephritis (Table 1). The patients with glomerular disease were taking angiotensin converting enzyme inhibitors or receptor blockers, and some patients with primary glomerulonephritis were taking steroids. Patients with acute kidney injury, heart failure, or acute infection were excluded. All data were collected via Korea Biobank Network in Chungbuk National University Hospital with informed consent of all patients and approved by hospital Institutional Review Board.

\section{Urine synaptopodin and slit diaphragm protein extraction}

Random spot urine samples were collected. The urine was centrifuged at 3,000 rpm for 10 minutes at $4^{\circ} \mathrm{C}$. Then, $200 \mu \mathrm{L}$ urine were removed from the supernatant, and an equal volume of protein extraction solution was added and mixed with a homogenizer at 15,000 rpm three times for 10 seconds each. After lysis for 30 minutes at $20^{\circ} \mathrm{C}$, the mixture was centrifuged at $13,000 \mathrm{rpm}$ at $4^{\circ} \mathrm{C}$ for $10 \mathrm{~min}$ utes. Using a spectrophotometer, the urine protein was measured by the Bradford method at $595 \mathrm{~nm}$.

\section{Western blot analysis}

Same $30 \mu \mathrm{g}$ of extracted proteins were loaded as $50 \mu \mathrm{L}$ in each well. Urine protein electrophoresis was performed using 10\% polyacrylamide-SDS gels and a SE 400 verti-

Table 1. Characteristics of the patients $(n=33)$

\begin{tabular}{lc}
\hline Characteristic & Value \\
\hline Age, yr & $49 \pm 16.5$ \\
Male sex & $16(48.5)$ \\
Mean serum creatinine, mg/dL & $2.30 \pm 1.76$ \\
Mean urine albumin/Cr ratio & $4.85 \pm 3.52$ \\
\hline Diagnosis of glomerulonephritis & \\
Diabetic nephropathy & $12(36.4)$ \\
Membranous nephropathy & $8(24.2)$ \\
IgA nephropathy & $6(18.2)$ \\
Minimal change diseases & $3(9.1)$ \\
\hline Lupus nephritis & $2(6.1)$ \\
FSGS & $1(3.0)$ \\
Diffuse proliferative GN & $1(3.0)$ \\
\hline
\end{tabular}

Values are presented as mean \pm SD or number (\%). IgA, immunoglobulin A; FSGS, focal segmental glomerulosclerosis; GN, glomerulonephritis. 
cal slab gel electrophoresis unit. Then, the bands were transferred to a polyvinylidene fluoride membrane using a TE 62 tank transfer unit. The primary antibodies for synaptopodin, podocalyxin, podocin, and nephrin were from Santa Cruz Biotechnology (Dallas, TX, USA), and the monoclonal anti- $\beta$-actin used as a loading control was from Sigma-Aldrich (St. Louis, MO, USA). The band density was measured three times each using Multi Gauge V3.1 software (Fuji Photo Film Co. Ltd., Tokyo, Japan), and $\beta$-actin was used for the reference density.

\section{Statistical analysis}

All data were analyzed using IBM SPSS statistics version 20 (IBM Co., Armonk, NY, USA). Association between urine synaptopodin and serum creatinine were explored by Kendal's tau correlation. Multiple regression analysis was used to find variable which effect to increase serum creatinine, and Kruskal-Wallis rank sum test was used to compare urine synaptopodin excretion among the dif- ferent estimated glomerular filtration rate (eGFR) group of the patients. To compare the excretion of urine podocyte-related proteins between diabetes and glomerulonephritis patients, the Mann-Whitney test was used, as the data did not show a normal distribution according to the Kolmogorov-Smirnov and Shapiro-Wilk tests. All data are presented as means and standard deviations and the statistical significance level was set at $p<0.05$ (2-tailed).

\section{RESULTS}

\section{Patient characteristics}

Among the 33 patients, 16 were men (48.5\%). The mean age was $49 \pm 16.5$ years, the mean serum creatinine was $2.30 \pm 1.76 \mathrm{mg} / \mathrm{dL}$, and the mean urine albumin/creatinine ratio was $4.85 \pm 3.52$. Diagnosis of each glomerular disease were diabetic nephropathy (36.4\%), membranous nephropathy (24.2\%), IgA nephropathy (18.2\%), minimal

Table 2. Factors associated with renal function impairment

\begin{tabular}{lcc}
\hline \multirow{2}{*}{ Variable } & \multicolumn{2}{c}{ Serum creatinine } \\
\cline { 2 - 3 } Diabetes & $\beta$ & $p$ value \\
\hline Urine albumin/Cr ratio & -0.46822 & 0.1020 \\
Urine nephrin/actin & -0.10445 & 0.1256 \\
Urine podocin/actin & 0.03766 & 0.7756 \\
Urine podocalyxin/actin & -0.06822 & 0.1518 \\
Urine synaptopodin/actin & -0.02464 & 0.1273 \\
Adjusted $R^{2}$ & 0.38101 & 0.0008 \\
Fvalue & & 0.5285 \\
\hline
\end{tabular}

${ }^{\mathrm{a}} \mathrm{p}<0.001$ with multiple regression analysis.

Table 3. Urine podocyte proteins in diabetic nephropathy and glomerulonephritis

\begin{tabular}{lccc}
\hline Variable & Diabetic nephropathy $(\mathrm{n}=12)$ & Glomerulonephritis $(\mathrm{n}=21)$ & $p$ value ${ }^{\mathrm{a}}$ \\
\hline Age, yr & $62.3 \pm 10.0$ & $41.9 \pm 14.9$ & $<0.001$ \\
Serum Cr, mg/dL & $3.65 \pm 1.73$ & $1.53 \pm 1.27$ & $<0.001$ \\
Urine albumin/Cr ratio & $5.10 \pm 3.53$ & $4.71 \pm 3.60$ & 0.726 \\
Urine podocyte proteins & & & 0.048 \\
Urine nephrin/actin & $1.85 \pm 1.28$ & $1.59 \pm 3.55$ & 0.033 \\
Urine podocalyxin/actin & $41.80 \pm 36.24$ & $25.04 \pm 39.62$ & 0.009 \\
Urine podocin/actin & $8.51 \pm 9.00$ & $3.67 \pm 7.02$ & $<0.001$ \\
\hline Urine synaptopodin/actin & $7.68 \pm 5.61$ & $2.56 \pm 3.11$ & $<$ \\
\hline
\end{tabular}

Values are presented as mean $\pm \mathrm{SD}$.

${ }^{a}$ Mann-Whitney test was used for comparing two groups. 
change disease (9.1\%), lupus nephritis (3.0\%), and diffuse proliferative glomerulonephritis (3.0\%) (Table 1).

\section{Urine synaptopodin excretion is strongly correlated with serum creatinine}

The serum creatinine level showed a strong correlation with age, as expected $(r=0.483, p<0.01)$. However, urine albumin/Cr ratio was not correlated with any of the podocyte proteins. Among the podocyte proteins, only synaptopodin showed strong association with serum creatinine by multivariate regression analysis, but the diabetes did not affect to serum creatinine elevation $(p<0.001$ ) (Table 2). Also, urine synaptopodin was strongly correlated with serum creatinine comparing with other podocyte proteins by Kendal's tau correlation test $(r=0.429, p<0.01)$ (Fig. 1). There was no significant statistic correlation between serum creatinine and slit diaphragms; nephrin $(r=-0.020, p=0.910)$, podocin $(r$ $=0.181, p=0.314)$, and podocalyxin $(r=0.180, p=0.316)$. Comparing three groups of the patient by eGFR, urine synaptopodin was markedly elevated in the patients with lower eGFR ( $p=0.0006)$ (Fig. 2 ).

\section{Urine slit diaphragm proteins and serum $\mathrm{Cr}$ were increased in the diabetic kidney disease}

The patients with diabetes were older $(p<0.001)$ and had a higher serum creatinine level $(p<0.001)$ compared

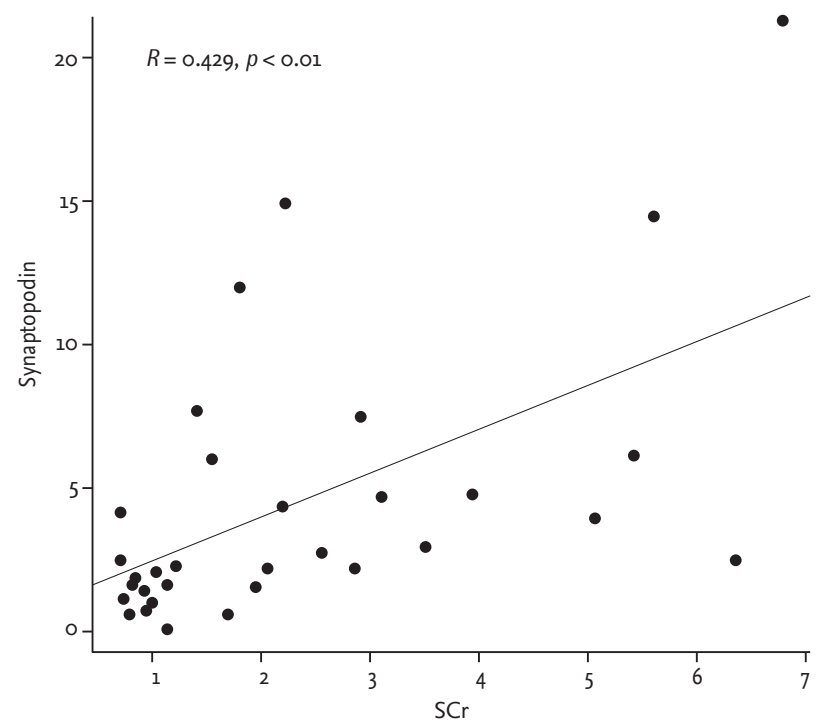

Figure 1. Urine synaptopodin excretion showed significant statistical correlation with serum creatinine ( $\mathrm{SCr}$ ) in all the patients with glomerulopathy. with those with glomerulonephritis. There was no statistical difference in the urine albumin/Cr ratio between the patients with diabetes and glomerulonephritis $(p=$ o.73) (Table 3). Comparing diabetes with glomerulonephritis, the urine nephrin/actin band density was $1.85 \pm$ 1.28 vs. $1.59 \pm 3.55(p=0.048)$, respectively, podocin/actin was $8.51 \pm 8.99$ vs. $3.67 \pm 7.02(p=0.009)$, podocalyxin/ actin was $41.80 \pm 36.24$ vs. $25.04 \pm 39.62(p=0.033)$, and synaptopodin/actin was $7.68 \pm 5.61$ vs. $2.56 \pm 3.11(p<0.001)$ (Table 3). Urine slit diaphragm proteins were increased in patients with diabetes, and synaptopodin showed the greatest significant difference (Fig. 3). The reference

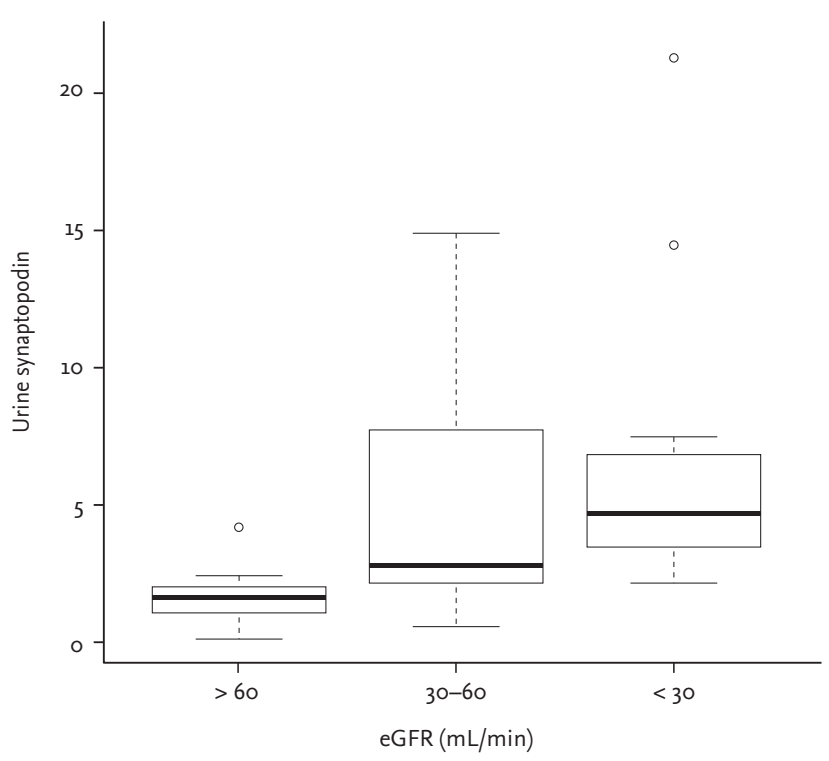

Figure 2. Amount of urine synaptopodin is significantly increased in the patients with decreased estimated glomerular filtration rate (eGFR) by Kruskal-Wallis rank sum test ( $p=$ $0.0006)$.

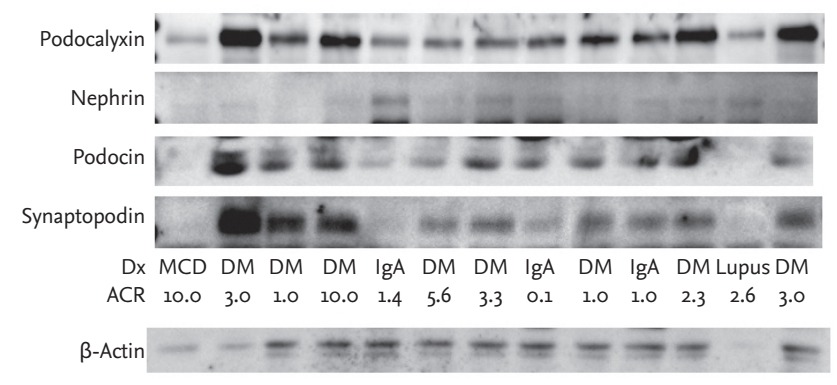

Figure 3. Western-blotting of urine podocyte proteins. Amount of urine albumin did not show any correlation with urine podocyte proteins. Dx, diagnosis; MCD, minimal change disease; IgA, immunoglobulin A; ACR, alumin/creatinine ratio. 
$\beta$-actin band density did not differ significantly between the diabetics and non-diabetics $(p=0.26)$.

\section{DISCUSSION}

Proteinuria is an important marker and prognostic factor of diabetic kidney disease progression, and overt proteinuria indicates advanced vascular damage affecting the glomeruli in diabetic nephropathy. In glomerulonephritis without diabetes, the amount of urine protein is also an important predictor of disease progression, and most proteinuria develops as a result of glomerular basement membrane damage related to slit diaphragm proteins. However, diabetic nephropathy is sometimes associated with normoalbuminuria and suggests microvascular damage that developed without slit diaphragm injury [8]. In this study, we found that the amount of urine albumin excretion did not correlate with any urine podocyte protein excretion, and renal function.

Podocyte injury in diabetic nephropathy can cause slit diaphragm destruction, and urinary slit diaphragm proteins may be markers of podocyte injury in diabetic nephropathy [5]. Podocyte nephrin expression is decreased in diabetes, and the urinary nephrin level is significantly correlated with urine albumin excretion in diabetic nephropathy [9]. Although renal biopsy is the most accurate diagnosis for any kind of glomerular disease, it so invasive that cannot be examined frequently. We tried to find a potential marker of podocyte damage, which is a major cause of glomerular disease progression regardless of proteinuria. We postulated that the slit diaphragm protein loss was related to urinary albumin excretion and attempted to evaluate podocyte loss by measuring the urinary excretion of synaptopodin, which is part of the podocyte cytoskeleton that regulates cytoskeletal motility. Interestingly, only urine synaptopodin showed greatest statistical correlation with serum creatinine elevation in all kinds of glomerular disease. Therefore, urine synaptopodin might reflect podocyte destruction, because synaptopodin is located in the foot process of the podocyte cytoskeleton.

Serum creatinine elevation is the most widely used marker of glomerular disease progression, and treatment to reduce proteinuria slows the decline in the GFR
[10]. Also, diabetic kidney disease shows worse clinical outcome than non-diabetic kidney disease [11]. In our study, renal function of the diabetes was worse than other glomerulonephritis. However, we found the elevated serum creatinine was not affected by diabetes via multiple regression analysis (Table 2). We found that urine synaptopodin excretion was strongly correlated with the elevation in serum creatinine comparing with other slit diaphragm proteins, but other slit diaphragm proteins did not affect renal function impairment by multiple regression analysis. Therefore, the amount of urinary synaptopodin excretion is increased by renal function impairment which likely reflects podocyte loss, indicating irreversible glomerular injury in advanced kidney disease. Interestingly, the other slit diaphragm proteins showed no correlation with renal impairment.

There are some limitations to this study. First, the study population was too small and lack of the patients with heavy proteinuria and normal renal function with diabetes. As we compared groups with similar levels of proteinuria, most of the patients with diabetic kidney disease with marked proteinuria had advanced kidney failure. The diabetic kidney disease patients might have generated more podocyte-specific proteins due to advanced kidney failure. Further studies on diabetics with heavy proteinuria and mild renal impairment might help elucidate the change in podocyte-specific proteins in diabetic kidney disease. Second, it was difficult to measure podocyte-specific proteins in urine samples, as there was no standard measurement method. We tried to quantify the proteins by Western blotting, but there was no standard control. We used $\beta$-actin to adjust measuring proteins to avoid band density error in repeated loading, as $\beta$-actin is found in many kinds of cells and commonly used as a loading control of cell integrity or protein degradation in Western blot analysis. Third, in this study, $\beta$-actin band density was different (Fig. 3), because urinary actin excretion depends by excretion of all kinds of urine cellular components including podocyte. We supposed that the patient with low actin and high slit diaphragm proteins might has higher excretion of detached podocyte proteins, and the patient with high actin and low podocyte protein might has lower podocyte protein excretion and large amount of urinary epithelial components. Interestingly, only synaptopodin band showed significant high density with or with- 
out actin adjustment. Also, there was no difference in $\beta$-actin excretion and was not correlated with amount of urine proteins in this study, but it is still not clear what the best control is for measuring urine proteins in Western blot analysis. If there is new method for measuring urinary podocyte proteins, we can find proper marker detecting podocyte damage.

However, we think this study might be a good example to reveal the importance of urine synaptopodin as a podocyte damage in advanced glomerulonephritis. If there are further studies which investigate urine synaptopodin in normal or mild renal impairment could expect the prognosis of glomerular disease with or without proteinuria.

In conclusion, the amount of urine synaptopodin excretion was increased in advanced kidney disease regardless of urine albumin excretion and it was significantly correlated with the serum creatinine elevation. We suggest that the urine synaptopodin level can predict glomerular damage independently of urine albumin excretion.

\section{KEY MESSAGE}

1. Urine synaptopodin excretion showed strong correlation with serum creatinine elevation in advanced glomerulonephropathy.

2. Amount of urine albumin excretion was not associated with urine podocyte protein excretion.

3. Urine synaptopodin could be an important marker of podocyte damage in glomerulonephropathy.

\section{Conflict of interest}

No potential conflict of interest relevant to this article was reported.

\section{Acknowledgments}

The research reported on this paper is supported by 2012 Chungbuk National University research grant.

The authors thank Tae-Young Heo in the Statistical
Analysis and Consulting Center in Chungbuk National University for thoughtful consulting of the statistics.

\section{REFERENCES}

1. Rutherford WE, Blondin J, Miller JP, Greenwalt AS, Vavra JD. Chronic progressive renal disease: rate of change of serum creatinine concentration. Kidney Int 1977;11:62-70.

2. Fornoni A. Proteinuria, the podocyte, and insulin resistance. N Engl J Med 2010;363:2068-2069.

3. Toyoda M, Najafian B, Kim Y, Caramori ML, Mauer M. Podocyte detachment and reduced glomerular capillary endothelial fenestration in human type 1 diabetic nephropathy. Diabetes 2007;56:2155-2160.

4. Yu D, Petermann A, Kunter U, Rong S, Shankland SJ, Floege J. Urinary podocyte loss is a more specific marker of ongoing glomerular damage than proteinuria. J Am Soc Nephrol 2005;16:1733-1741.

5. Zheng M, Lv LL, Ni J, et al. Urinary podocyte-associated mRNA profile in various stages of diabetic nephropathy. PLoS One 2011;6:e20431.

6. Asanuma K, Yanagida-Asanuma E, Faul C, Tomino Y, Kim K, Mundel P. Synaptopodin orchestrates actin organization and cell motility via regulation of RhoA signalling. Nat Cell Biol 2006;8:485-491.

7. Faul C, Donnelly M, Merscher-Gomez S, Chang YH, Franz S, Delfgaauw J, et al. The actin cytoskeleton of kidney podocytes is a direct target of the antiproteinuric effect of cyclosporine A. Nat Med 2008;14:931-938.

8. Pugliese G. Updating the natural history of diabetic nephropathy. Acta Diabetol 2014;51:905-915.

9. Jim B, Ghanta M, Qipo A, et al. Dysregulated nephrin in diabetic nephropathy of type 2 diabetes: a cross sectional study. PLoS One 2012;7:e36041.

10. Lewis EJ, Hunsicker LG, Clarke WR, et al. Renoprotective effect of the angiotensin-receptor antagonist irbesartan in patients with nephropathy due to type 2 diabetes. $\mathrm{N}$ Engl J Med 2001;345:851-860.

11. Byun JM, Lee CH, Lee SR, et al. Renal outcomes and clinical course of nondiabetic renal diseases in patients with type 2 diabetes. Korean J Intern Med 2013;28:565-572. 